\title{
Iterative Availability Assessment Approach for Multi-Feeder Industrial Energy System Sections
}

\author{
Tom Van Acker, Graduate Student Member, IEEE \\ Dirk Van Hertem, Senior Member, IEEE \\ Research Division Electa-ESAT \\ University of Leuven \\ Leuven, Belgium \\ Email: tom.vanacker@esat.kuleuven.be
}

\begin{abstract}
Industrial energy systems are predominantly radial systems, designed to supply energy services to industrial processes. Given the high interruption costs of industrial processes, industrial energy system operators often opt to include backup feeders to enable redundancy, i.e., to quickly restore supply in case of failure of the normally-closed feeders. Depending on the type of redundancy, the flow changes following redundancy activation, resulting in alteration of the stress on the feeder components and consequently the failure probability of the feeders. To this end, the concept of competing risk is introduced to account for this dependability between the section feeders. Additionally, for sections with active redundancy, which results in bidirectional dependency, an iterative procedure is introduced which updates the failure weights of the competing risks. Finally, two numerical illustrations are provided. The first numerical illustration shows the functionality of the iterative approach, whereas the second numerical illustration compares three different sections with respect to their total system cost. The latter numerical illustration also shows that unavailability risk depends on the outage duration and consequently influences the best section choice.
\end{abstract}

\section{INTRODUCTION}

An industrial energy system (IES) supplies energy to industrial processes within an industrial site. Adequate energy supply requires that the quality limits prescribed by the industrial processes are satisfied. Different energy vectors have different quality indicators, e.g., power system indicators include voltage, current and frequency, whereas flow system indicators include pressure and flow rate. If the IES state does not cause a violation of the quality limits prescribed by a certain industrial process, then the IES is said to be available for that industrial process, i.e., able to perform as required [1]. On the other hand, the IES becomes unavailable for a specific industrial process when the prescribed limits of that process are violated by the IES state, leading to an interruption of that process. Several surveys, including [2], [3], have shown that the financial losses due to interruption of industrial consumers significantly exceed those of other consumers, e.g., residential or commercial. Additionally, these surveys have shown that the financial loss greatly depends

This research is funded by a Ph.D. Fellowship of the Flemish Agency for Innovation \& Entrepreneurship in cooperation with BASF Antwerp NV (Grant No. 150257).

The ELECTA research group of KU Leuven is a founding member of the EnergyVille research lab. on three characteristics describing the quality limit violation causing the industrial process interruption: 1) cause, which quality limit was violated; 2) duration, how long was the quality limit violated; and 3) time, when was the quality limit violated.

An IES operator should balance the cost of quality against the value of quality when designing, maintaining and operating its energy system. This entails minimizing the sum of its utility cost and unavailability risk. The utility cost is the cost of designing, maintaining and operating the IES. The unavailability risk is defined as the product of the probability with the severity of IES unavailability; the latter is usually expressed as the cost representative of the cost representative of the financial losses due to IES unavailability. Inaccurate assessment of the unavailability results in an over- or underestimation of the unavailability risk, which in turn leads to over- or underinvestment in the IES, resulting in a total system cost which is not minimal. Reducing the modeling error is instrumental for improved design, maintenance and operation of the IES closer to its minimal total cost. To this end, an availability assessment method is presented for multifeeder IES sections enabled to take the following aspects into account:

- state probabilities in function of time $t$ and state sojourn time $\varphi$;

- incorporation of failure heterogeneity [4] as well as perfect and minimal corrective maintenance [5];

- incorporation of failure dependency due to active and passive redundancy;

- general time-homogeneous transition rates.

The basic structure of an IES is radial. However, a set of back-up feeders can be included to restore supply in case of failure of a normally-closed feeder. An IES section is defined as a collection of IES components which have a direct dependency due to recoverability actions following any failure of the included components. Following this definition, back-up feeders must be included in the section for which it provides redundancy for its normally-closed feeders. Examples of such multi-feeder sections: $s_{5}, s_{6}$ and $s_{9}$, are included in Fig. 1, where the back-up feeders are depicted in gray. Sections $s_{5}$ and $s_{9}$ are examples of active redundancy [6] and section $s_{6}$ of 


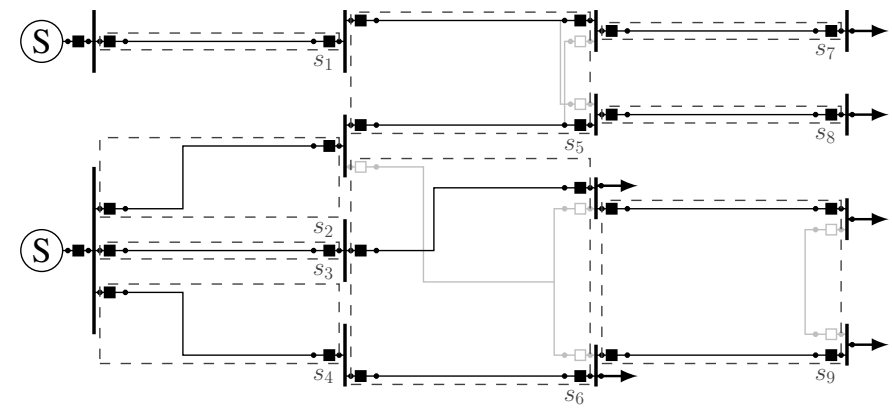

Fig. 1. Typical topology of an IES, where: node $(\bullet)$, feeder $(\bullet \bullet-)$, source ( (S) • ), open/closed interruptor ( $\square \bullet /(\bullet \bullet \bullet)$ ), industrial process

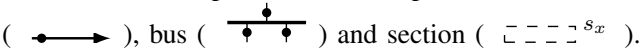

standby redundancy [7]. Utilizing a back-up feeder following the failure of a normally-closed feeder may alter the flow through the remaining active section feeders, as well as the back-up feeder itself. Changes in the flow through a feeder alters the stress on, and consequently the failure probability of that feeder. Direct incorporation of this dependability in a stochastic process, which also considers the age of the feeders, would introduce an age variable for each normallyclosed and back-up feeder, increasing the problem dimension and consequently its computational complexity. In order to avoid this, this paper presents an approach that decouples the problem, reducing the computational complexity whilst still incorporating the dependability between the different section feeders. The paper is organized as follows: section II defines the mathematical framework of the proposed approach. Section III presents two numerical illustrations. The first numerical illustration shows the functionality of the iterative approach, whereas the second numerical illustration compares three different sections with respect to their total system cost. Section IV concludes the paper.

\section{Mathematical Framework}

A section is composed of a set of feeders. In order to reduce computational complexity, the availability of each separate feeder is assessed using the method presented in the first subsection. The method is extended in order to take the dependability between the feeders into account. Additionally, this method is nested in an iterative approach to update the dependability between the feeders until the method converges.

\section{A. Feeder Availability Assessment Method}

The process governing the feeder availability is represented by a state transition diagram, which in turn is modeled as a directed graph $(\mathcal{S}, \mathcal{T})$. The sets $\mathcal{S}$ and $\mathcal{T}$ respectively describe a set of states $\left(i, \varphi_{i}\right)$ and transitions $i j$ going from $\left(i, \varphi_{i}\right)$ to $\left(j, \varphi_{j}\right)$. The former is the union of two disjoint subsets $\mathcal{N}$, a singular set containing the normal operation state, and $\mathcal{P}$, composed of all post-failure states. Any state $\left(i, \varphi_{i}\right)$ is discrete with respect to the state $i$ itself, but continuous with respect to its state sojourn time $\varphi_{i}$. The state sojourn time $\varphi_{i}$ describes the time spend in the state before leaving the state. In the context of this paper, the normal operation state sojourn time is best conceptualized as the age of the feeder, where any post-failure state sojourn time represents the duration of the recoverability or maintenance action(s) performed in the considered state. For example, if the feeder is in a state $s$ where it is being replaced, then $\varphi_{s}$ represents the time it took to replace the feeder. Moreover, these post-failure state sojourn times are representative of the duration of the associated unavailability of that state. The probability of occupying a state $i$ for a certain sojourn time $\varphi_{i}$ at a time $t$ is defined as $p_{i}\left(t, \varphi_{i}\right)$. Within the state transition diagram $(\mathcal{S}, \mathcal{T})$, a set $\mathcal{C}$ is defined containing all simple cycles $c$ consisting of the normal operation state $n$ and a subset of post-failure states $\mathcal{P}_{c}$. A simple cycle is a closed sequence of states within a directed graph with no repetition of states or transitions, other than the starting state. In the context of this paper, a simple cycle $c$ represents a sequence of recoverability and maintenance actions within the state-transition diagram $(\mathcal{S}, \mathcal{T})$, including the corresponding failure, in order to restore a feeder to its normal operation state. For example, following a failure $f$ the resulting fault is cleared, after which perfect corrective maintenance is performed restoring the feeder to its normal operation state (Fig. 2). Reducing all simple cycles into two state cycles, i.e., the normal operation state and a merged state representative of all post-failure states of that respective cycle, and consequently integrating the expression for the merged state probabilities into that of the normal operation state probability, results in the following partial delay differential equation (1) with non-local boundary condition (2) for the normal operation state probability:

$$
\begin{aligned}
\frac{\partial p_{n}}{\partial t}+\frac{\partial p_{n}}{\partial \varphi_{n}}= & -\sum_{(f, c) \in \mathcal{R}} \lambda_{f}\left(t, \varphi_{n}\right) p_{n}\left(t, \varphi_{n}\right) \\
& +\sum_{(f, c) \in \mathcal{R}^{\mathrm{M}}}\left(f_{c} * \lambda_{f} p_{n}\right)\left(t, \varphi_{n}\right), \\
p_{n}(t, 0)= & \sum_{(f, c) \in \mathcal{R}^{\mathrm{P}}} \int_{0}^{\infty}\left(f_{c} * \lambda_{f} p_{n}\right)\left(t, \varphi_{f}\right) \mathrm{d} \varphi_{f},
\end{aligned}
$$

where the set $\mathcal{R}$ contains all reduced cycles $(f, c)$ with a corresponding failure $f$ and cycle $c$. The reduced cycle set $\mathcal{R}$ consists of two disjoint subset $\mathcal{R}^{\mathrm{M}}$ and $\mathcal{R}^{\mathrm{P}}$, respectively entailing minimal and perfect corrective maintenance at the end of the cycle $c$. Any reduced cycle $(f, c)$ is described by its failure rate $\lambda_{f}$ and cycle transition probability density function (pdf) $f_{c}$.

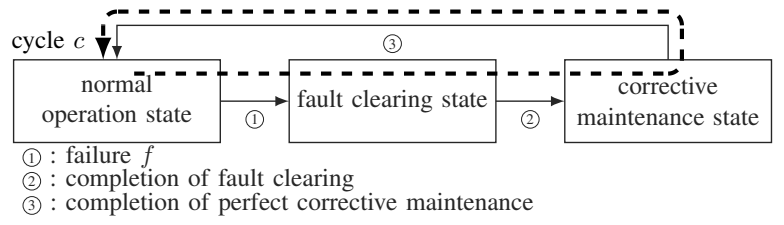

Fig. 2. Example of a cycle within a state-transition diagram $(\mathcal{S}, \mathcal{T})$ representing the feeder availability 
The problem structure of (1)-(2) permits the use of cohort strategy presented in [8] to discretize the solution space (Fig. 3). Each cohort variable $a \in \mathcal{A}$ links time $t$ and normal operation state sojourn time $\varphi_{n}$ using:

$$
t=\varphi_{n}+a, \quad \forall a \in \mathcal{A} .
$$

In the context of this paper, a cohort variable $a$ essentially groups the probability of a feeder in function of its age $\varphi_{n}$, which would have been equal to zero within a time frame $[a, a+\mathrm{d} a]$. Using (3), (1) and (2) reduce to a set of nonhomogeneous first order ordinary differential equations (4) and initial conditions (5):

$$
\begin{aligned}
\frac{\mathrm{d} p_{n, a}}{\mathrm{~d} \varphi_{n}}= & -\sum_{(f, c) \in \mathcal{R}} \lambda_{a, f}\left(\varphi_{n}\right) p_{n, a}\left(\varphi_{n}\right) \\
& +\sum_{(f, c) \in \mathcal{R}^{\mathrm{M}}} \sum_{x \in X_{c}} f_{c, x} \lambda_{a, f}\left(\varphi_{n}\right) p_{a-x, n}\left(\varphi_{n}\right), \quad \forall a \in \mathcal{A},
\end{aligned}
$$

$$
p_{n, a}(0)=\sum_{(f, c) \in \mathcal{R}^{\mathrm{P}}} \sum_{x \in X_{c}} \int_{0}^{\infty} f_{c, x} \lambda_{a, f}\left(\varphi_{n}\right) p_{a-x, n}\left(\varphi_{f}\right) \mathrm{d} \varphi_{f}, \forall a \in \mathcal{A},
$$

where for a fixed cohort variable step-size $\mathrm{d} a$, the set $X_{c}=$ $\left\{1, \ldots,\left\lceil\varphi_{m}^{\max } / \mathrm{d} a\right\rceil\right\}$ and $f_{c, x}=\int_{(x-1) \mathrm{d} a}^{x \mathrm{~d} a} f_{c}\left(\varphi_{m}\right) \mathrm{d} \varphi_{m}$.

After finding a numerical solution for the normal operation state probability $p_{n}$, it can be used to determine all post-failure state probabilities $p_{p}$. Any post-failure state $p$ can belong to multiple cycles $c$, each such combination is described by $f_{c, p}^{\mathrm{pre}}$ and $R_{c, p}$. For any cycle $c$, the former represents the convolutions of all transitions between the normal operation state $n$ and the considered post-failure state, excluding the failure transition. The latter represents the survival function corresponding to the subsequent transition away form the considered post-failure state $p$. Consequently, a post-failure state probability $p_{p}\left(t, \varphi_{p}\right)$ can be expressed as

$$
p_{p}\left(t, \varphi_{p}\right)=\sum_{(f, c) \in \mathcal{R}: c \in \mathcal{C}_{p}}\left(f_{c, p}^{\mathrm{pre}} * p_{f}\right)\left(t-\varphi_{p}\right) R_{c, p}\left(\varphi_{p}\right),
$$

where $p_{f}(t)=\int_{0}^{\infty} \lambda_{f}\left(t, \varphi_{n}\right) p_{n}\left(t, \varphi_{n}\right) \mathrm{d} \varphi_{n}$ represents the failure probability with respect to $t$ for a certain failure $f$.

\section{B. Iterative Method Incorporating Feeder Dependability}

A feeder can experience a set of failures $\mathcal{F}$, each of which can be linked to a weight $w_{f}(t)$, representing the probability of the feeder being in a state where a failure $f$ can occur at a time $t$. Using (3), $w_{f}(t)$ is expressed as $w_{f, a}\left(\varphi_{n}\right)$. The concept of competing risk, initially introduced in mathematical biology and recently adopted in a reliability context [9], [10], can be used to model the different feeder failures. Competing risks are said to be present when a subject is at risk of more than one mutually exclusive failure, and the occurrence of any one prevents any other from happening. Within the context of the cohort approach, this definition holds as a failure within a cohort characteristic results in the departure from that characteristic, and thus per definition no other failure can occur

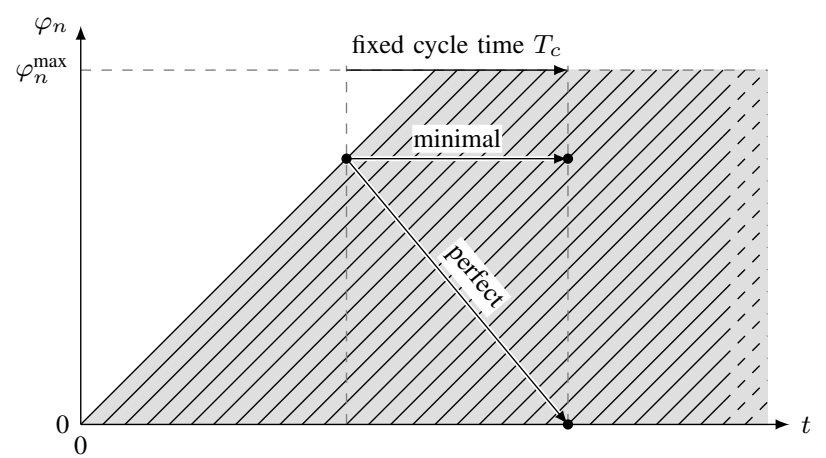

Fig. 3. Lexis diagram of the solution space. Under the assumption of $p_{n}(0,0)=1$, the grey area represents the solution space for the PDDE (1) and its non-local boundary condition (2), where $\varphi_{n}$ is limited to $\varphi_{n}^{\max }$, and the black lines represent the solution space for the set of ODE (??) and initial conditions (??).

on that characteristic. The cause-specific failure rate $\lambda_{a, f}\left(\varphi_{n}\right)$ is the instantaneous risk of failure $f$ at an operation time $\varphi_{n}$ within a characteristic $a$ given that the feeder has not failed up until an operation time $\varphi_{n}$. This is expressed as the specific failure pdf, weighted with $w_{f, a}\left(\varphi_{n}\right)$, divided by the overall complementary cumulative distribution function (ccdf):

$$
\lambda_{a, f}\left(\varphi_{n}\right)=\frac{w_{f, a}\left(\varphi_{n}\right) f_{a, f}\left(\varphi_{n}\right)}{1-\sum_{f \in \mathcal{F}} \int_{0}^{\varphi_{n}} w_{f, a}\left(\varphi_{n}\right) f_{a, f}\left(\varphi_{i}\right) \mathrm{d} \varphi_{i}} .
$$

In case of sections with build-in redundancy, the activation of these redundancies alters the stress on certain feeders of that section. Redundancy activation generally follows the failure of a normally-closed feeder. As a direct consequence, the failure weights $w_{f}(t)$ are the combination of the post-failure state probabilities of those feeders warranting this redundancy activation. Given the expression of the state probability with respect to time and sojourn time, the redundancy activation description can be significantly extended, allowing modeling closer to reality. For example, a redundancy is activated within one of two attempts: 1) after five minutes, success probability: $90 \%$; 2) after fifteen minutes, success probability: $50 \%$, whenever feeder $i$ is in a primary cleared state following a subset of failures. In case of standby redundancy, e.g., $s_{6}$ in Fig. 1, there only exists one-directional dependency, i.e., the back-up feeder's failure weights $w_{f}(t)$ depend on the availability of the normally-closed feeders but not the other way around. This means that the availability of the redundant feeder can be directly assessed following the availability assessment of the normally-closed feeders. However in case of active redundancy, e.g., $s_{5}$ in Fig 1, there exists twodirectional dependency, which means that the failure weights of one feeder depend on the state probabilities of another feeder and vice versa. This means that the availability of the feeders cannot be directly determined.

To this end, an iterative procedure (Fig. 4) is proposed which updates the failure weights each iteration until the method converges. Initially, all failure weights $w_{f}(t)$, except for the base failure weights, are set to zero, whereas the latter are set 


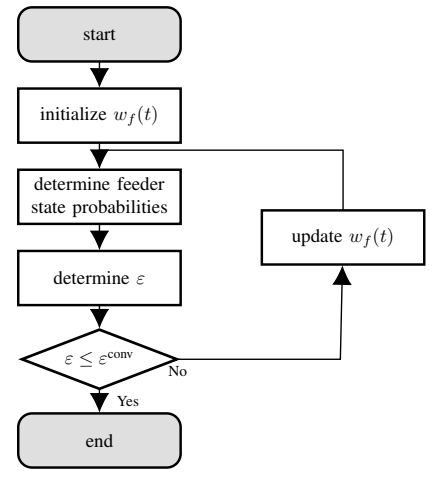

Fig. 4. Iterative method which updates the failure weights $w_{f}(t)$ until the method has converged based on the Fréchet distance $\varepsilon$ between the current and previous iteration's normal state probability $p_{n}(t)$.

to one. Consequently, the state probabilities are determined using these failure weights based on the strategy presented in II-A. After which, the Fréchet distance $\varepsilon$ [11] is determined between the normal operation probability $p_{n}(t)$ of the current and the previous iteration loop ${ }^{1}$. If the distance $\varepsilon$ exceeds the user-defined limit $\varepsilon^{\text {conv }}$, the failure weights are updated and the procedure is repeated, otherwise the method is considered to be converged.

\section{NUMERICAL ILLUSTRATIONS}

This section presents two numerical illustrations. The first numerical illustration shows the functionality of the proposed iterative strategy on a small test case, i.e., a two feeder section, each with two possible failures. The second numerical illustration compares three sections with respect to their total system cost, i.e., the sum of its utility cost and unavailability risk.

\section{A. Numerical Illustration I}

A section $s_{I}$ comprised of two feeders is proposed (Fig. 5a). Each feeder provides active redundancy for the other, i.e., upon failure of one feeder, the other feeder takes over in order to keep all industrial processes supplied. This is accomplished by either closing interruptor $b^{\text {left }}$ if the right feeder fails, or alternatively closing interruptor $b^{\text {right }}$ when the left feeder fails. Each feeder is consequently prone to two possible failures: 1) normal failure $\lambda_{n}$, i.e., when $b$ is open; 2) stress failure $\lambda_{s}$, i.e., when $b$ is closed. For the purpose of this test case, there is no delay in closing of the interruptor $b$ following a normal failure. Each of these failures require perfect corrective maintenance with their respective repair rate $\mu_{n}$ and $\mu_{s}$, resulting in the feeder state-transition diagram depicted in Fig. 5b. For the purpose of this test case, all transitions are modeled with underlying exponential pdf,

$$
f(t, \varphi)=\frac{w(t)}{\chi} \exp (-\varphi / \chi), \quad t, \varphi \geq 0 .
$$

Table I gives an overview of the necessary data for the considered transitions. The weights $w(t)$ of the failures $\lambda_{n}$

\footnotetext{
${ }^{1}$ This step is skipped for the first iteration.
}

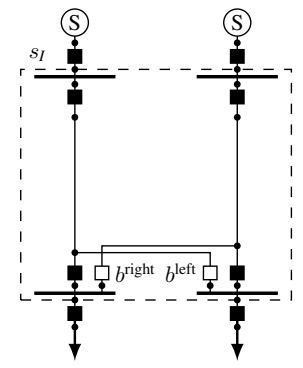

(a)

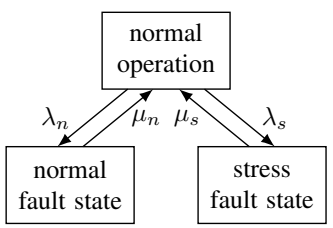

(b)
Fig. 5. Test section (5a) and associated feeder state-transition diagram (5b).

and $\lambda_{s}$ depend on the state probabilities of the other feeder and need to be determined through the presented iterative process. Initially, the normal failure weights $w_{n}(t)$ are set to one, whereas the stress failure weights $w_{s}(t)$ are set to zero. During the iterative process, the weights for iteration $i$ are updated based on the state probabilities of the previous iteration $i-1$. The weights $w_{n}(t)$ for the normal failure are equal to the sum of the normal operation state probability and stress fault state probability of the opposite feeder (9), whereas the weights $w_{s}(t)$ of the stress failure are equal to the normal fault state probability of the other feeder (10).

$$
\begin{aligned}
& w_{n, i}(t)=p_{n, i-1}(t)+p_{f, s, i-1}(t) \\
& w_{s, i}(t)=p_{f, n, i-1}(t)
\end{aligned}
$$

Given the symmetry of the two feeders transitions, only the results for the left feeder are presented. The iterative process needs five iteration using a user-defined limit $\varepsilon^{\text {conv }}$ equal to $10^{-8}$. Figure 6 shows the evolution of the normal operation state probability $p_{n}(t)$ for all five iterations. Throughout the iteration process, the normal operation state probability is sequentially over- and underestimated. The final normal operation state probability has a lower steady state value compared to the initial steady state variable. This is because in contrary to the initial iteration, the last iteration also considers the stress failure, which has a higher failure rate and a longer average repair time compared to the normal failure.

Section $s_{I}$ is unavailable with respect to the left industrial process, similar for the right industrial process given the section symmetry, in two scenarios: 1) the left feeder has experienced a stress failure which has not been repaired; 2) the left feeder has experienced a normal failure which has not been repaired and the right feeder is unavailable to take over. The probability of the former scenario is equal to the stress fault state probability of the left feeder $p_{f, s}^{\text {left }}$. However, determining the probability of the latter scenario is slightly more complex, as it essentially is a joint probability of two events A and B:

TABLE I

TRANSITION DATA FOR THE STATE-TRANSITION DIAGRAM (FIG. 5B).

\begin{tabular}{ccc|ccc} 
Symbol & $\chi[\mathrm{h}]$ & $w(t)$ & Symbol & $\chi[\mathrm{h}]$ & $w(t)$ \\
\hline$\lambda_{n}$ & $10^{4}$ & $*$ & $\mu_{n}$ & $10^{2}$ & 1.0 \\
$\lambda_{s}$ & $0.5 \cdot 10^{4}$ & $*$ & $\mu_{s}$ & $2 \cdot 10^{2}$ & 1.0
\end{tabular}




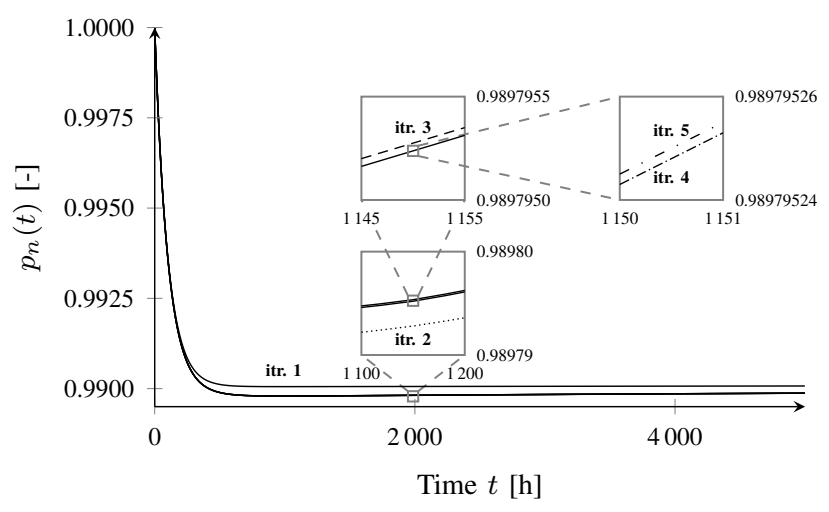

Fig. 6. Iterations (itr.) of the normal operation state probability, where itr. 1: solid line, itr. 2: dotted line, itr. 3: dashed line, itr. 4: densely dashdotted line and itr. 5: loosely dashdotted line.

$A$ - the left feeder has experienced a normal failure which has not been repaired;

$B$ - the right feeder is unavailable to take over.

The joint probability of two events can be determined using

$$
\mathbb{P}(A \cap B)=\mathbb{P}(B) \cdot \mathbb{P}(A \mid B),
$$

where $\mathbb{P}(B)$ equals the addition of the two fault states of the right feeder, i.e., $p_{f, n}^{\text {right }}$ and $p_{f, s}^{\text {right }} \cdot \mathbb{P}(A \mid B)$ is the conditional probability of the left feeder having experienced a normal failure which has not been repaired given the unavailability of the right feeder to take over. However, as events A and $\mathrm{B}$ are independent, the joint probability $\mathbb{P}(A \cap B)$ can be expressed as

$$
\mathbb{P}(A \cap B)=\mathbb{P}(B) \cdot \mathbb{P}(A) .
$$

\section{B. Numerical Illustration II}

1) Test System and Test Case: For the second numerical illustration, three section topologies are compared based on their total system cost, which is the sum of the utility cost and unavailability risk. Figure 7 presents the different section topologies and the corresponding feeder state-transition diagrams. In the first section $s_{I I-A}$, there is no build-in redundancy. This allows to assess the availability of each feeder separately, as there is no dependability between the feeders ${ }^{2}$. The second section $s_{I I-B}$ uses the feeders themselves as active redundancy options when either of them fail. As shown in the previous numerical illustration, the availability assessment of such a section requires the presented iterative approach. The third section $s_{I I-C}$ has an additional feeder as a standby redundancy option which takes over whenever any of the normally-closed feeders fails. Compared to the second section, a failure of one normally-closed feeder does not cause any stress on the other normally-closed feeder of the third topology. The only dependency in the third section is of the outer feeders

${ }^{2}$ Given the definition for a section presented in section I, the two feeders should be considered as individual sections. However, in order to enable straightforward comparison between the three considered sections $s_{I I-A}-s_{I I-C}$, the two feeders are treated as one section. on the inner feeder, but not the other way around. To this end, no iterative procedure is necessary for the third section, however the dependability requires a sequential assessment of the feeder state probabilities. First, the state probabilities of the outer feeders are determined, after which the inner feeder state probabilities are determined using the state probabilities of the outer feeders to find its failure weights.

In order to have a fair comparison between the three topology options, it is assumed that the feeder failures are the same under similar conditions, i.e., a normal failure $\lambda_{n}$ when the feeder is loaded for $50 \%$ and a stress failure $\lambda_{s}$ when the feeder loaded for $100 \%$. Each of these failures require minimal corrective maintenance, which is assumed to be without cost. All transitions are modeled with an underlying exponential pdf. Table I gives an overview of the failure and repair data. In order to compare the total cost of the three sections, both their utility cost and unavailability risk needs to be determined. For the purpose of this numerical illustration the utility cost for the simulated period $(T=5000 h)$ is set to be $2.0 \mathrm{M} \$$ for the first section, $2.5 \mathrm{M} \$$ for the second section and $3.0 \mathrm{M} \$$ for the third section. The marginal outage cost $m(\varphi)$ with respect to outage duration $\varphi$ is modeled as a shifted sigmoid function (13) with a cost multiplier $c$ and a duration multiplier $d$. The former allows to scale the amplitude of the function, where the latter allows to stretch the function with respect to duration $\varphi$.

$$
m(\varphi)=\frac{c}{1+\exp ((-\varphi+6) / d)}, \quad \varphi \geq 0
$$

2) Results and Discussion: As the utility cost is given for all three sections, determining their total cost comes down to determining their unavailability risk $U$ over the simulation period $T$. The latter can be determined using

$$
U=\sum_{f \in \mathcal{F}} \int_{0}^{T}\left(p_{f} *\left(m R_{f}\right)\right)(t) \mathrm{d} t,
$$

where, $p_{f}(t)$ represents the failure probability in function of time $t, m(\varphi)$ represents the marginal outage cost (13) and $R_{f}(\varphi)$ represents the survival function of the corresponding fault state. The latter is equal to the ccdf of the repair action used to leave the corresponding fault state. Given the symmetry of the sections, (15)-(17) give the failure probabilities for all three sections $s_{I I-A}-s_{I I-C}$, where the second section is the only one where the normally-closed feeders can experience both a normal failure $\lambda_{n}$ and a stress failure $\lambda_{s}$,

$$
\begin{aligned}
p_{f}^{\mathrm{A}}(t) & =2 \int_{0}^{\infty} \lambda_{n}\left(t, \varphi_{n}\right) p_{n}^{\mathrm{A}}\left(t, \varphi_{n}\right) \mathrm{d} \varphi_{n}, \\
p_{f}^{\mathrm{B}}(t) & =2\left(p_{f, n}^{\mathrm{B}}(t)+p_{f, s}^{\mathrm{B}}(t)\right) \int_{0}^{\infty} \lambda_{n}\left(t, \varphi_{n}\right) p_{n}^{\mathrm{B}}\left(t, \varphi_{n}\right) \mathrm{d} \varphi_{n} \\
& +2 \int_{0}^{\infty} \lambda_{s}\left(t, \varphi_{n}\right) p_{n}^{\mathrm{B}}\left(t, \varphi_{n}\right) \mathrm{d} \varphi_{n}, \\
p_{f}^{\mathrm{C}}(t) & =2\left(p_{f, n}^{\mathrm{C}}(t)+p_{f, s}^{\mathrm{C}}(t)\right) \int_{0}^{\infty} \lambda_{n}\left(t, \varphi_{n}\right) p_{n}^{\mathrm{C}}\left(t, \varphi_{n}\right) \mathrm{d} \varphi_{n} .
\end{aligned}
$$




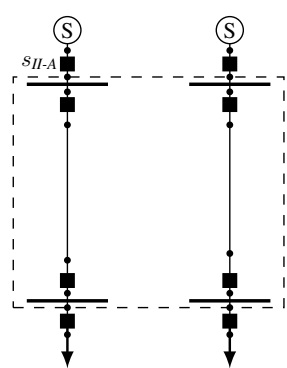

(a)

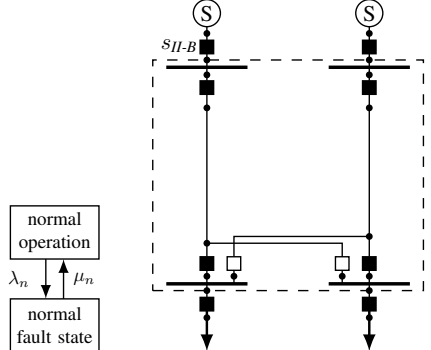

(c)

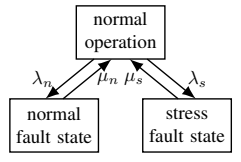

(d)

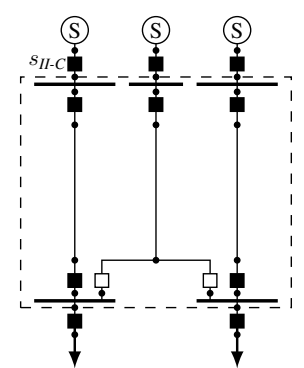

(e)

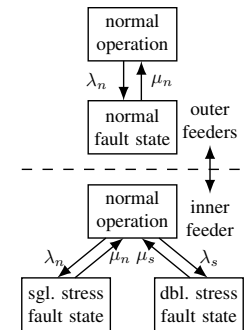

(f)

Fig. 7. Test systems and accompanying state-transition diagrams. A first section (7a) has no build-in redundancy, resulting in the feeder state-transition diagram (7b). The second section (7c) uses the feeders themself as redundancy, resulting in the feeder state-transition diagram (7d). The third section (7e) has a separate feeder which can be used for redundancy, resulting in two possible state-transition diagrams (7f), one for the inner feeder and one for the outer feeders.

Using these equations, the unavailability risk can be determined and consequently the total cost of each section. Figure 8 gives an overview of the best section choice for a range of both the cost multiplier $c$ and duration multiplier $d$. For certain ranges of the cost multiplier $c$, the best section choice is independent of the duration multiplier $d$. However for certain ranges of the cost multiplier $c$, the duration multiplier $d$ plays a role in determining the best section choice. In both cases, i.e., the limit between sections $s_{I I-A}$ and $s_{I I-B}$ as well as between section $s_{I I-B}$ and $s_{I I-C}$, the difference in cost multiplier $c$ between the outer limits of the duration multiplier $d$ is a factor two.

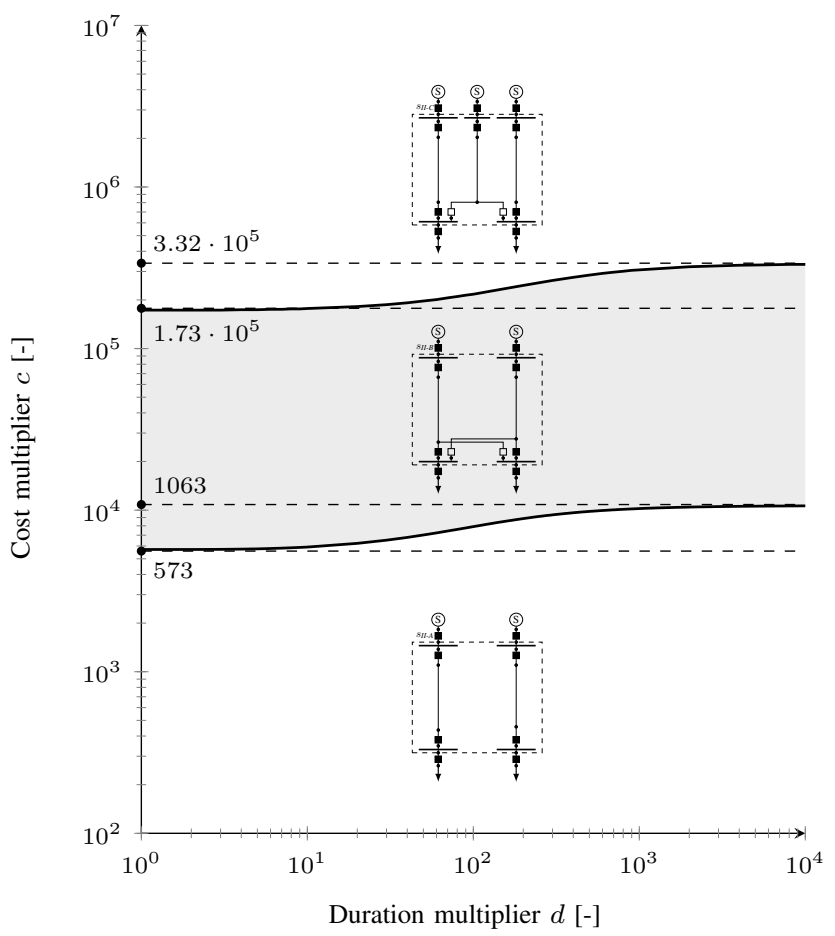

Fig. 8. Section with the lowest total cost in function of cost multiplier $c$ and duration multiplier $d$.

\section{CONCLUSION}

In order to avoid an increase in mathematical complexity when assessing the availability of multi-feeder IES sections with active redundancy, an iterative procedure is proposed in this paper allowing to decouple the assessment of the individual feeders. To this end, the concept of competing risks is used. Two numerical illustrations are introduced, a first numerical illustration showcases the method and its quick convergence. In the second numerical illustration, three different section topologies are compared with respect to their total cost. It shows that the duration dependability of the marginal outage cost plays a significant part in the choice of the best section around the limit between the two sections.

\section{REFERENCES}

[1] International Electrotechnical Commission (IEC), "Availability, <of an item $>$," 2015. [Online]. Available: http://www.electropedia.org/iev/iev.nsf/display?openform\&ievref=192-01-23 [Accessed: Jan. 4, 2018]

[2] M. J. Sullivan, T. Vardell, and M. Johnson, "Power interruption costs to industrial and commercial consumers of electricity," IEEE Trans. Ind. Appl., vol. 33, no. 6, pp. 1448-1458, 1997.

[3] G. H. Kjølle, K. Samdal, B. Singh, and O. A. Kvitastein, "Customer costs related to interruptions and voltage problems: methodology and results," IEEE Trans. Power Syst., vol. 23, no. 3, pp. 1030-1038, 2008.

[4] J. H. Jürgensen, L. Nordström, and P. Hilber, "A review and discussion of failure rate heterogeneity in power system reliability assessment," in 2016 Int. Conf. Probabilistic Methods Appl. to Power Syst., Beijing, 2016, pp. 1-8.

[5] H. Wang, "A survey of maintenance policies of deteriorating systems," Eur. J. Oper. Res., vol. 139, no. 3, pp. 469-489, 2002.

[6] International Electrotechnical Commission (IEC), "Active Redundancy," $2015 . \quad$ [Online]. Available: http://www.electropedia.org/iev/iev.nsf/display?openform\&ievref=192-10-03 [Accessed: Jan. 4, 2018]

[7] - "Standby Redundancy," 2015. [Online]. Available: http://www.electropedia.org/iev/iev.nsf/display?openform\&ievref=192-10-04 [Accessed: Jan. 4, 2018]

[8] L. Abia and J. Lopes-Marcos, "Runge-Kutta methods for age-structured population models," Appl. Numer. Math., vol. 17, no. 1, pp. 1-17, 1995.

[9] E. Keedy and Q. Feng, "Reliability analysis and customized preventive maintenance policies for stents with stochastic dependent competing risk processes," IEEE Trans. Reliab., vol. 62, no. 4, pp. 887-897, 2013.

[10] K. Sabri-Laghaie and R. Noorossana, "Reliability and maintenance models for a competing-risk system subjected to random usage," IEEE Trans. Reliab., vol. 65, no. 3, pp. 1271-1283, 2016.

[11] S. Har-Peled and B. Raichel, "The fréchet distance revisited and extended," ACM Trans. Algorithms, vol. 10, no. 1, pp. 1-22, 2014. 